\title{
Tunable hetero-assembly of a plant pseudoenzyme-enzyme complex
}

\section{Authors}

Irina V. Novikova ${ }^{1}$, Mowei Zhou ${ }^{1 *}$, Chen $\mathrm{Du}^{2,3}$, Marcelina Parra ${ }^{4}$, Doo Nam Kim ${ }^{5}$, Zachary L.

VanAernum $^{2,3}$, Jared B. Shaw ${ }^{1}$, Hanjo Hellmann ${ }^{4}$, Vicki H. Wysocki ${ }^{2,3}$ and James E. Evans ${ }^{1,4 *}$

\section{Affiliations}

${ }^{1}$ Environmental Molecular Sciences Laboratory, Pacific Northwest National

Laboratory, Richland, WA, USA

${ }^{2}$ Department of Chemistry and Biochemistry, The Ohio State University, Columbus, OH 43210, USA.

${ }^{3}$ Resource for Native Mass Spectrometry Guided Structural Biology, The

Ohio State University, Columbus, OH 43210, USA.

${ }^{4}$ School of Biological Sciences, Washington State University, Pullman,

WA, 99164, USA

${ }^{5}$ Biological Science Division, Pacific Northwest National Laboratory,

Richland, WA, USA

Corresponding Authors E-mail: james.evans@pnnl.gov,

irina.novikova@pnnl.gov,mowei.zhou@pnnl.gov

\section{Keywords}

Vitamin $\mathrm{B}_{6}$, PLP, PDX, cell-free expression, co-expression, Native Mass Spectrometry, cryo-EM, heterocomplex, electrostatics, pseudoenzyme 


\section{Abstract}

Pseudoenzymes have emerged as key regulatory elements in all kingdoms of life despite being catalytically non-active. Yet many factors defining why one protein is active while its

4 homolog is inactive remain uncertain. For pseudoenzyme-enzyme pairs, the similarity of both

5 subunits can often hinder conventional characterization approaches. In plants, a pseudoenzyme

6 PDX1.2 positively regulates vitamin $\mathrm{B}_{6}$ production by association with its active catalytic

7 homologs such as PDX1.3 through an unknown mechanism. Here we used an integrative experimental approach to learn that such pseudoenzyme-enzyme pair associations result in hetero-

9 complexes of variable stoichiometry, which are unexpectedly tunable. We also present the atomic

10 structure of the PDX1.2 pseudoenzyme as well as the population averaged PDX1.2-PDX1.3

11 pseudoenzyme-enzyme pair. Finally, we dissected hetero-dodecamers of each stoichiometry to

12 understand the arrangement of monomers in the hetero-complexes and identified symmetry-

13 imposed preferences in PDX1.2-PDX1.3 interactions. Our results provide a new model of

14 pseudoenzyme-enzyme interactions and their native heterogeneity.

\section{Introduction}

Pseudoenzymes are homologs of enzymes that are catalytically deficient or inactive. Prior research

18 from select protein families of kinases and phosphatases has shown that many pseudoenzymes retain the

19 structural fold of their catalytically active partners and function as protein interaction modules ${ }^{1}$. Structural

20 studies were the key tools in exploring and clarifying the molecular mechanisms of such systems and they

21 uncovered their roles as allosteric regulators, scaffolds, molecular switches and substrate competitors ${ }^{2-4}$.

22 These pseudoenzymes have to be diligently targeted, or anti-targeted (i.e. avoid intervention), for desirable

23 outcomes in drug design or bioengineering applications so as to not be indiscrimately treated as their active

24 homologs. To understand pseudoenzyme roles on a wider scale, genomic studies now show that 
pseudoenzymes account for $5-10 \%$ of the proteome and are associated in specialized signaling networks across all life domains ${ }^{5}$. However, the high structural similarities between pseudoenzymes and canonical enzymes create new experimental challenges in classical molecular and biochemical characterization approaches for unraveling the underlying mechanism. This is the case for the present study, which is focused around pseudoenzyme PDX1.2, a regulator of vitamin $\mathrm{B}_{6}$ biosynthesis in plants.

Vitamin $\mathrm{B}_{6}$ functions as a cofactor for over a hundred enzymatic processes and also provides an important defense mechanism against oxidative stress and pathogen infection ${ }^{6-10}$. Plants are able to synthesize vitamin $\mathrm{B}_{6}$ (PLP) de novo by two enzymes - PDX1 and PDX2 (Pyridoxine Biosynthesis 1 and $2)^{6,11}$. In particular, in Arabidopsis thaliana, there are three homologs of PDX1, designated as PDX1.1, PDX1.2 and PDX1.3, and one homolog of PDX2 ${ }^{11}$. Of these, PDX1.1, PDX1.3 and PDX2 are all catalytically active enzymes while PDX1.2 has been shown to be an inactive variant ${ }^{11}$. In vivo experiments showed that PDX1.2 expression is upregulated by heat and oxidative stress and coincides with a boost in

37 vitamin $\mathrm{B}_{6}$ synthesis, suggesting a positive regulatory impact ${ }^{12-14}$. PDX1.2 was first proven to interact with its catalytic PDX1.1 and PDX1.3 proteins in vivo and to form complexes of high molecular weight similar to its catalytic homologs ${ }^{15}$. Recombinant co-expression of PDX1.2 with either PDX1.1 or PDX1.3 showed dodecameric hetero-complexes ${ }^{13}$.

PDX1.2 protein resists crystallization protocols while PDX1.3 homo-complexes and PDX1.2/PDX1.3 hetero-complexes are compatible with macromolecular crystallography ${ }^{16}$. However, 43 unlike PDX1.3 homo-complexes, the crystals of PDX1.2/PDX1.3 hetero-complexes sufferred from a

44 statistical disorder, where the positions of individual proteins were not distinguished despite exhaustive 45 approaches used ${ }^{16}$. The ambiguity in the data prohibited the determination of the assembly mechanism but 46 an interhexamer assembly mechanism was proposed as the likely cause of interaction where one hexameric 47 ring of active PDX1.1 (or PDX1.3) stacks on top of a hexamer ring of inactive PDX1.2 (Fig.1a) to create a 48 final hetero-dodecameric state ${ }^{13,16}$. Herein, using an integrated biochemical and structural approach 49 combining cell-free protein synthesis technology, native mass spectrometry (native MS), and cryo-electron 
microscopy (cryo-EM), we show that PDX1.2 and PDX1.3 form hetero-dodecamers through intrahexamer association of two symmetric or similar hexamers with varying but tunable stoichometry.

\section{Results}

\section{Cell-free expression of PDX homo/hetero-complexes}

We employed a cell-free protein expression pipeline using wheat germ extract ${ }^{17-19}$ to provide the

56 closest translational environment for the production of these plant proteins in terms of folding and post-

57 translational modifications. The "open-format" of a cell-free platform provided us with an opportunity for precise stoichiometric control of protein co-expression (Fig.1b) by varying the amounts of corresponding DNA templates, not easily achieved by cell-based protein expression methods. We prepared two DNA plasmids constructs (one for PDX1.2 and one for PDX1.3), and combined them in specific molar ratios to achieve the desired protein output in the co-expression conditions (Fig.S1).

In the initial cell-free translation experiments, we supplemented all the translation reactions with

63 fluorophore-labeled lysine-charged tRNA as recently described ${ }^{19}$. This procedure allowed detection of

64 newly-synthesized proteins in the crude mixture with no need for purification. For co-expression, different DNA template ratios $(9: 1,3: 1,1: 1,1: 3$ and 1:9) were tested to establish that the molar ratio of expressed proteins can be controlled in a precise fashion. For instance, by in-gel quantification, the co-expression conditions 9:1, 3:1 and 1:1 correspond to protein product compositions of 3:1, 1:1 and 1:3 (Fig.1c). The same samples were also analyzed under native gel conditions where all co-expressed samples traveled faster than individual PDX1.2 homo-complexes but slower than PDX1.3 homo-complexes. In addition, the bands

70 for co-expression complexes appeared significantly wider in size suggesting the presence of multiple

71 stoichiometric dodecamer species. The mobilities of these samples also appeared to be dependent on the

72 protein composition where a higher proportion of PDX1.3 in the co-expressed sample caused faster 73 migration of the hetero-complex, and the opposite was true for higher PDX1.2 content. The variable hetero-

74 complex migration on the gel and the thickness of the bands do not support the previously proposed 
interhexamer assembly mechanism ${ }^{16}$ where a defined single band of a single stochiometric ratio of 6:6 would be expected. Instead, PDX1.2 and PDX1.3 likely form mixed hetero-complexes.

We also verified that co-expression was essential for the PDX1.2 and PDX1.3 hetero-complex formation, supporting previous discoveries ${ }^{13,16}$. Mixing the two separately expressed and purified proteins post-translationally did not produce detectable amounts of hetero-complexes on native PAGE (Fig. S2a).

80 The same co-expression experiments were conducted using active PDX1.1 enzyme (instead of PDX1.3)

81 and PDX1.2, and identical pseudoezyme-enzyme assembly behavior was observed (Fig.S2b). Because 82 PDX1.3 is the dominant homolog in $A$. thaliana ${ }^{20,21}$ and was previously found to be more impacted enzymatically by the presence of PDX1.2 ${ }^{13}$, we have limited our further structural survey to PDX1.2 and

PDX1.3.

\section{Tunable stochiometry and activity of PDX hetero-complexes}

For further structural characterization, we scaled-up the synthesis and then purified select samples: PDX1.2 and PDX1.3 homo-complexes and hetero-complexes at the co-expression conditions 9:1, 3:1 and and PDX1.3, respectively (Fig. S5), which are consistent with their monoisotopic theoretical masses of

$92 \quad 37339.6 \mathrm{Da}$ and $36405.3 \mathrm{Da}$. Although approximately 50\% of the protein N-termini were found to be

93 acetylated, no preferential acetylation profile was detected suggesting these post-translational modifications

94 are unlikely to play a role in PLP biosynthesis regulation (Fig. S5-S6).

95 While the native PAGE results indicated the presence of mixed co-complexes in each co-expression 96 condition, the technique did not have the resolution to define the stoichiometry. We therefore employed

97 native MS, where we electrosprayed the proteins under nondenaturing conditions in $200 \mathrm{mM}$ ammonium 98 acetate solution. The intact 12 mers were detected around $m / z$ of 9000 , carrying $\sim 50$ positive charges (Fig.

99 S7). All species in the co-expressed samples were resolved and the stoichiometry could be accurately 100 determined from the measured mass, as shown by the native MS spectrum of co-complex 9:1 in Fig. 2a. 
101 From the spectra, mass distributions of all species in the sample can be deconvoluted by combining the

102 intensities across charge states (Fig. 2b). As expected, the PDX1.2 and PDX1.3 samples showed

103 predominantly homo-12mers with molecular weights of $449 \mathrm{kDa}$ and $437 \mathrm{kDa}$, respectively. Each co-

104 expressed sample generated 6-8 hetero-complexes of variable stoichiometry that were all dodecameric and

105 influenced by the initial plasmid DNA ratio input to the cell-free reaction. For example, the 9:1 co-

106 expression condition resulted in a pool of hetero-complexes with PDX1.2 representing the major protein

107 constituent and spanning observed stoichiometry values in the $6: 6$ to $12: 0$ range $(6: 6,7: 5,8: 4,9: 3,10: 2$,

$10811: 1$ and 12:0). For the 3:1 co-expression condition, there is a shift to the 3:9-11:1 stoichiometry range,

109 while the 1:1 co-expression condition was limited to the 0:12 - 8:4 stoichiometry range (Fig. 2b). The

110 observed stoichiometry values were consistent with the amounts of proteins produced in each sample (see

111 Fig.1c and Fig. S3). By varying the input DNA ratio (and thus protein ratios), the full range of co-complexes

112 from $0: 12$ to $12: 0$ stoichiometry can be sampled, demonstrating the tunability of these protein-protein

113 associations.

Interestingly, a significant amount of free monomer and dimer species were observed for PDX1.3

115 containing complexes in the low $m / z$ region $<6000$ (Fig. S7). In the co-expressed samples, the relative

116 abundance of PDX1.3 monomers and dimers was also found to be positively correlated with the ratio of

117 PDX1.3 in the hetero-complexes. In contrast, PDX1.2 appeared to be more stable under the same

118 experimental conditions. We confirmed that $200 \mathrm{mM}$ ammonium acetate did not cause noticeable protein

119 unfolding or disassembly in solution prior to MS (Fig. S8). Previous native MS studies have shown that

120 positively charged proteins may interact with silanol groups on borosilicate glass surface at sub-micron

121 sized emitter tips, resulting in supercharging and unfolding during electrospray ${ }^{22}$. In addition, supercharging

122 typically makes complexes easier to dissociate in vacuo ${ }^{23,24}$. Therefore, the dissociation of PDX1.3

123 containing 12mers might be due to unique charging properties of PDX1.3 that are distinct from PDX1.2.

124 To understand how the PDX1.2:PDX1.3 ratio in hetero-assembly affects their enzymatic

125 performance, we employed a diagnostic assay where PLP is used as a cofactor molecule for apo-enzyme

126 chemical transformations, the products of which were detected by a colorimetric approach (Fig.2c). Under 
127 these assay conditions with saturating substrate amounts, the specific activity of active enzyme PDX1.3

128 was determined to be $793 \mu \mathrm{moles} / \mathrm{min} / \mathrm{mg}$ protein. As expected, pseudoenzyme PDX1.2 was found to be

129 inactive and displays a slightly inhibitory effect. When proteins are co-expressed under conditions 3:1 and

130 1:1, the presence of PDX1.2 hetero-complex positively impacts PLP synthesis with an increase of up to

$131120 \%$ in relative enzymatic activity. However, at 9:1, where $75 \%$ of the total protein is PDX1.2, the hetero-

132 complex displays a decline in relative activity to $67 \%$ of that observed for PDX1.3 homo-12mer. Yet, the

133 measured activities represent a sum of PDX1.2 and PDX1.3. If these relative enzymatic activities are

134 normalized based on the amount of catalytically active PDX1.3 in each co-expressed sample, the positive

135 regulatory impact of PDX1.2 is observed for every condition (Fig. 2d). The higher ratio of PDX1.2

136 incorporation showed higher impact on PDX1.3 catalytic activity with the corresponding outputs: 3 -fold

137 increase for co-expression condition 9:1, 2.4-fold increase for 3:1 and 1.5-fold increase for 1:1 (Note that

138 co-expression conditions 9:1, 3:1, and 1:1 correspond to protein ratios $3: 1,1: 1$, and 1:3, respectively).

\section{Lateral symmetry within the seemingly stochastic assembly}

To resolve the structures of the purified complexes we employed single particle cryo-EM. The

146 the expected dodecameric fold known for catalytically active PDX1.1 and PDX1.3. The final 3D volume

147 was reconstructed at $3.2 \AA$ resolution, and a PDX1.2 homology model was then docked and real space 148 refined (Fig. S10, Table S1). Residues 29-288 were clearly resolved while N- (residues 1-28) and C-

149 terminal (residues 289-313) regions appear rather flexible due to the lack of associated densities in the

150 volume. We also obtained a high-resolution cryo-EM dataset for the 9:1 co-expression complex (Fig. S11).

151 A well-defined two-ring fold seemed visually identical to the PDX1.2 homo-12mer. Various heterogenous, 
non-uniform and local refinements did not sort out the potential subclasses, which is not surprising due to

153 variability of the stoichiometry of the co-expressed species, the similarity of PDX1.2 and PDX1.3 folds,

154 and the small difference $(<1 \mathrm{kDa})$ between their monomer molecular weights. As a result of the lack of

155 distinct subclasses, the 9:1 co-expression complex particle dataset was processed as a single 3D class, and

156 non-uniform refinement yielded the final map. Both the PDX1.2 and PDX1.3 homomers can be fitted

157 independently with high validation scores (Fig. S12, Table S1) to any of the monomer positions - similar

158 to the result reported recently for X-ray structure ${ }^{14}$. In other words, the two different subunits cannot be

159 easily distinguished in the hetero-complex, suggesting a stochastic hetero-assembly.

To better understand the distribution of the subunits within the hetero-complexes, we mass isolated

161 individual heteromers of each stoichiometry and subjected them to surface induced dissociation (SID) in a

modified mass spectrometer ${ }^{25}$. The charge states of the proteins were reduced by adding triethylammonium

acetate in solution, which suppressed unfolding upon activation and allowed more informative dissociation

products to be obtained in $\operatorname{SID}^{26}$ (Fig. S13). A representative SID spectrum for the 6:6 hetero-complex is

shown in Fig. 3a. Collision of the precursor ions into the surface under controlled laboratory frame collision

energy $(5.6 \mathrm{keV})$ in SID caused a rapid increase in an internal energy for the $12 \mathrm{mer}$, leading to the

167 dissociation into subcomplexes. Our previous studies have shown that subcomplexes generated in SID are

168 often from dissection of the weakest interfaces and are reflective of the native quaternary structure of protein

169 complexes $^{27,28}$. In contrast, collision-induced dissociation (commercially available as higher-energy

170 collisional dissociation, $\mathrm{HCD}$, in the instrument used here) results in activation of the 12 mer via multiple

171 low-energy collisions and, at most, only resulted in stripping of unfolded monomers at the maximum

172 collision energy (10.5 keV) (Fig. S14). Among the released species in SID, the most abundant was the 6mer

173 (Fig. 3b). The other species such as 4mers and 8mers were also detected but at lower abundance. The major

174 products, 6mers, are likely the result of horizontal cleavage along the interface of two 6mer rings (Fig. 3c).

175 Another minor pathway of two vertical cleavages while maintaining the lateral interactions may have

176 generated the 4 mer+8mer pair and also contributed partially to the signal of 6 mers. We suspect the 5 mers 
177 in both spectra may partially originate from secondary dissociation of 6mers and are not from direct

178 cleavage of the 12 mers because of the low abundance of the complementary 7 mers. Herein, we focus on

179 the major pathway for structural elucidation of the hetero-assemblies.

The deconvoluted SID spectra show a relatively monodispere stoichiometric distributions in the

released 6mers. For example (Fig. 3b), $70 \%$ of the 6 mers from the $6: 6$ heteromer had the stoichometry of

$1823: 3$ while the remainder were found to be $2: 4$ and 4:2. This suggests that the $6: 612$ mers are formed in either

183 the [3:3 and 3:3] or [2:4 and 4:2] combination of PDX1.2:PDX1.3. For 7:5 heteromer with odd numbers

184 of each protein in the 12 mer, 6mers with stochiometry of both 3:3 and 4:2 were released at similar

185 intensities. The most abundant 6 mers released by SID from other 12 mers all have half of the stoichiometry

186 of the 12mer precursors (Fig. S15). We estimated the abundances of the 6mers generated only from the

187 major pathway (see methods for details) and plotted the relative abundances of the different stoichiometry

188 as a function of the 12 mer precursor stoichiometry in Fig. $3 \mathrm{~d}$. The narrow distribution of the stoichiometry

189 of the 6mer rings strongly favors a lateral symmetry in the 12mers. If PDX1.2 and PDX1.3 were distributed

190 stochastically, a wide variety of stochiometries should be observed in the released 6mers (Fig. S16).

\section{Similar protein folds but inverted electrostatic surface potentials}

198 to K166 (PDB:5lnr) were all aligned with our structure of PDX1.2 and overlaid for comparison (Fig. 4a).

199 The PDX1.3 enzyme employs a lysine relay mechanism where all catalytic action happens within a single

200 domain using two catalytic sites $\mathrm{P} 1$ and $\mathrm{P} 2^{29,30}$. Two key residues K98 and K166 are at the heart of this 
process where they trap the substrates, covalently tether the intermediates and then shuttle the product out.

We found that the overall monomer fold of PDX1.2 is very similar to PDX1.3, and the best fit for PDX1.2

203 is to the X-ray structure of PDX1.3-PLP, which has the P1 site unoccupied.

Importantly, the overall monomer fold of PDX1.2 is identical to PDX1.3 at the outer shell where proper higher order protein interactions with neighboring subunits need to be preserved (Fig. 4a). The conservation pressure on those amino acids, which are involved in quaternary interactions, appears not to

207 be specific for $A$. thaliana and is imposed on many plant organisms (Fig. S17). Thus, the maintaince of 208 PDX1.2 quaternary contacts with PDX1.3 is very important for pseudoenzyme functional performance. 209 Major differences between PDX1.2 and PDX1.3 are observed in the area surrounding the catalytic site P1. 210 Specifically, the $\mathbf{\alpha 2}$ ' helix and $\mathbf{3 6}-\mathbf{\alpha 6}$ loop are significantly altered in PDX1.2. The $\boldsymbol{\alpha 2}$ ' helix, present in 211 PDX1.3, is absent in PDX1.2 due to the amino acid truncation in that region and is substituted by a loop 212 that points slightly outwards. The amino acid changes in the $\mathbf{3 6 - \alpha 6}$ loop, including an amino acid insertion, 213 create a slight kink in PDX1.2 architecture towards the center of the 12mer, resulting in the potential 214 weakening/aborting of the phosphate binding at this site. Key lysine residues K98 and K166 which form 215 covalent adducts with the intermediates of PLP formation in PDX1.3 are mutated to R100 and Q168 216 respectively in PDX1.2. While R100 is positioned similarly to K98 and can potentially form the imine bond 217 with R5P as well, Q168 in PDX1.2 (in place of K166 in PDX1.3) points towards the P1 site similar to the 218 intermediate state of the PLP synthesis instead of pointing to P2 as observed in the priming PDX1.3-R5P 219 state. The same positional occupancy of Q168 was assigned to the composite assignment of Q168 and K166 220 in the crystallized PDX1.2-PDX1.3 co-complex where individual protein positions were not 221 distinguished ${ }^{16}$. Such structural alterations in the P1 site in PDX1.2 are in agreement with the experimental 222 data, showing the loss of catalytic potential. Unlike P1, the catalytic site P2 in PDX1.2 appears to be 223 undisturbed except for the mutation of the key residue K166 to Q168. 
there is no clear mechanism about how PDX1.2 can positively regulate PDX1.3. We did not find any significant differences in the interfaces between subunits (Fig. S18). Hydrophobicity plots of PDX1.2 and PDX1.3 did not show any difference between the positions of polar and hydrophobic residues (Fig. S19).

Molecular modeling using Rosetta showed no obvious impact of PDX1.2 on the stability of the complex.

Surprisingly, the Rosetta calculation estimated that PDX1.3 packs more tightly than PDX1.2 and has more

231 favorable sum of interactions and interface stablization (Table S2). Therefore, other regulatory

232 contributions that cannot be captured by current computational methods are likely present (e.g. long-range

233 surface electrostatics changes).

Interestingly, the most stunning differences were found when comparing the electrostatic surface potentials between the two (Fig. 4b). The surface potential of outer and inner regions of the PDX1.2 12mer, which spans the P1 and P2 sites, appears inverted relative to PDX1.3, where negative charges replace the positively charged regions. The difference in charge based on the structures was also consistent with the apparent lower stability of PDX1.3 than PDX1.2 in native MS as mentioned earlier. The positive electrostatic surface potential of PDX1.3 could induce charging and unfolding in electrospray, giving rise to significant dissociation in the native MS spectra.

\section{Discussion}

Pseudoenzymes represent a largely uncharted territory with much to learn about the additional layer

244 of regulatory control they impose in nature. They are now known for allosteric regulation and scaffolding 245 through conserved binding interfaces or domains like their active enzyme homologs ${ }^{5}$. Yet, technical 246 challenges exist in probing such pesudoenzyme-enzyme interactions due to extremely high structural 247 similarity, as exemplified by the PDX1.2-PDX1.3 pseudoenzyme-enzyme pair detailed in this work.

248 A single particle cryo-EM approach was used to solve the structure of PDX1.2, a protein known 249 not to crystallize. We found that the PDX1.2 fold closely mimics the fold of PDX1.3, a common trend 250 observed for pseudoenzymes involved in allosteric regulation of their catalytic counterparts. PDX1.2 also 251 displays an altered catalytic site P1 which appears perturbed in concordance with its lack of activity, while 
the P2 site remains largely unchanged. In the studies described herein, we were able to accurately control the stoichiometry of hetero-complexes using cell-free expression and characterize them using native MS.

254 To our surprise, the catalysis can be tuned by shifting pseudoenzyme-enzyme stoichiometry, a discovery that was not reported previously for any known pseudoenzyme-enzyme pair today.

Additionally, the tunable nature of PDX1.2 and PDX1.3 co-complexes strongly disfavors the 257 previously proposed assembly model that promoted interaction through homomeric 6mer rings (Fig.1a).

258 Our native MS and SID data demonstrated that PDX1.2 co-assembly with its catalytic partner PDX1.3 is

259 largely based on stochastic subunit incorporation at different locations but with some degree of symmetry

260 (Fig.5a). Driven by the molar ratios of individual components during co-expression conditions, the entire

261 range of all possible stoichiometry combinations (from 12:0 to 0:12) has been recorded.

Another intriguing finding is the inverted surface charge of PDX1.2 compared to PDX1.3. It has

been shown that surface charge-charge interactions can be redesigned to increase protein stability ${ }^{31}$. As

PDX1.2 expression is induced under heat stress, the switch in the electrostatic surface potential might serve concordance with the observed mechanism of assembly and amino acid conservation, we hypothesize that the most profound effect would be on the catalytic site P2. Site P2 is located at the edge of the PDX1.3 subunit and shares two subunit-subunit interfaces: side-by-side I1' within the hexamer ring and bottom-

271 bottom I3 at the ring-ring interface (Fig.5b). We constructed several PDX1.2-PDX1.3 assembly models

272 around the catalytic site P2 of PDX1.3 by swapping subunits in the aligned homo-12mer structures (Fig.5c-

273 f). Lateral PDX1.2 incorporation can create a local supercharging effect on the $\mathbf{3 4 - \alpha 4}$ loop, which could

274 potentially act on the nitrogen atom of its pyridoxine ring (Fig.5d). This supercharging effect could promote

275 imine bond breaking between PLP and K166, help in the translocation and assist with the exit of the final

276 product and thus increase the rate of reaction turnover. The supercharging of the same loop could also be 277 possible when PDX1.2 shares a bottom-bottom interface (Fig.5e). The positioning of PDX1.2 on the 
bottom also slightly weakens the charge state around the phosphate binding site in $\alpha 4$. Both side and bottom consequent positioning of PDX1.2 (Fig.5f) could explain enhanced PDX1.3 activity in the hetero-complex and the benefit of having a pseudoenzyme that hetero-associates with active PDX1.3 in the 12mer.

While our current data cannot determine precisely which interfaces in the hetero-interaction are

282 favored, the constructed models strongly suggest the benefit of such interactions for enzyme activity. Given

283 the inverted electrostatic surface potentials of the two proteins, attractive forces between opposite charges

284 may favor the symmetry in such hetero-interactions. We thus propose that PDX1.2 and PDX1.3 monomers

285 are placed in an alternating manner within the hetero-complex so that the hetero-interactions can be

286 maximized. This is supported by our observation that homo-subcomplexes were not released in SID for

287 most hetero-complexes (Fig. S15) - meaning the same protomers do not prefer to cluster within the 12 mer.

288 Such positioning strategy empowers a simple, tunable and gentle approach to accelerate vitamin $\mathrm{B}_{6}$ 289 production by active enzymes such as PDX1.3 and PDX1.1.

Although the monomer positions within individual hetero-complexes cannot be structurally

291 resolved by either cryo-EM or X-ray crystallography, native MS provided accurate determination of

292 stoichiometry and SID revealed the lateral symmetry in the subunit arrangement. Ongoing development in

293 native MS instrumentation and the gas-phase protein behaviors would allow more structural information to

294 be extracted from such experiments in the near future. Our study shows that complementing high resolution

295 structural biology techniques such as cryo-EM with native MS offers new ways to understand heterogenous

296 pseudoenzyme-enzyme interactions that cannot be elucidated by single techniques alone.

Methods

Plasmid construction. Gene sequences of PDX1.2 and PDX1.3 were sourced as described

300 previously $^{19}$. The clones were amplified by PCR to include the purification tag and then sub-cloned using

301 Gibson Assembly in the designated vector $\mathrm{pEU}$ for the cell-free protein synthesis ${ }^{19,32}$. All reagents for PCR

302 and Gibson reactions such as the Q5 Hot Start High-Fidelity 2X Master Mix and Gibson Assembly Master

303 Mix were acquired from New England Biolabs. DNA primers were from ThermoFisher Scientific. For 
expression of PDX1.2 and PDX1.3 proteins, two vectors were constructed: pEU_3XFLAG_PDX1.2 and

pEU_3XFLAG_PDX1.3, where both genes were fused with the 3XFLAG purification tag on their N-

terminus (the sequences of synthesized proteins are in Fig. S1). The sequences of all plasmids used in this

307 study were verified by Sanger sequencing (MCLAB).

Cell-free expression/co-expression and purification of PDX proteins. Protein synthesis was carried out using Wheat Germ Protein Research Kits WEPRO7240 from CellFree Sciences. For co-

311 expression, stock plasmids were prepared containing various amounts of pEU_3XFLAG_PDX1.2 and

312 pEU_3XFLAG_PDX1.3. For example, in the case of co-expression at 9:1: $9 \quad \mu 1$ of $1 \mu \mathrm{g} / \mu 1$

313 pEU_3XFLAG_PDX1.2 was mixed with $1 \mu \mathrm{l}$ of $1 \mu \mathrm{g} / \mu \mathrm{l}$ pEU_3XFLAG_PDX1.3 before the transcription

314 step. The MINI-scale protein synthesis in the presence of fluorophore-charged lysine tRNA was conducted

315 with the following conditions: (i) for transcription, $1 \mu \mathrm{lof} 1 \mu \mathrm{g} / \mu \mathrm{l}$ stock plasmid was mixed with $9 \mu \mathrm{l}$ of

316 Transcription premix solution (1x Transcription buffer, $2.5 \mathrm{mM} \mathrm{NTP,} 8 \mathrm{U} / \mu 1 \mathrm{SP} 6$ polymerase, $8 \mathrm{U} / \mu 1 \mathrm{RNase}$

317 inhibitor) and left to incubate at $37^{\circ} \mathrm{C}$ for 4 hours, (ii) for translation, $2 \mu$ of the resulting mRNA was mixed

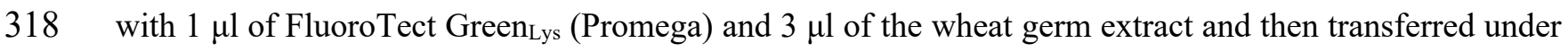

$31950 \mu 1$ of the translation buffer (1x SUB-AMIX SGC) within a 96-well half-area plate. The translations were

320 conducted overnight at $15^{\circ} \mathrm{C}$ and away from light in the vibration-free setting on an Eppendorf

321 ThermoMixer C.

The MAXI-scale protein synthesis was performed using the robotic system - Protemist DT II from

323 CellFree Sciences. All steps were performed at $4^{\circ} \mathrm{C}$ and at $140 \mathrm{rpm}$. The buffer exchange step of the crude

324 extract (in order to remove dithiothreitol, DTT) was skipped. A total of $600 \mu 1$ of ANTI-M2 affinity resin

325 (50\% suspension, pre-equilibrated in 1xTBS buffer, Sigma) was used per each $6 \mathrm{ml}$ translation reaction.

326 Protein binding to the resin was carried out for 1 hour. The resin was subsequently washed three times with

$3272.5 \mathrm{ml}$ of 1xTBS buffer for $5 \mathrm{~min}$ each time. The protein products were eluted twice with the $1 \mathrm{ml}$ Elution

328 Buffer (1xTBS buffer, $100 \mu \mathrm{g} / \mathrm{ml}$ 3XFLAG peptide, $4 \mathrm{mM}$ DTT) each time for $30 \mathrm{~min}$. Proteins were

329 further concentrated and buffer-exchanged to the Storage Buffer (1xTBS with 4 mM DTT) using $30 \mathrm{kDa}$ 
Amicon Ultra-2 centrifugal filter units. The concentrated samples were flash-frozen in liquid nitrogen in 10

$\mu \mathrm{l}$ aliquots and then transferred to $-80^{\circ} \mathrm{C}$ for storage.

General PAGE electrophoresis conditions employed in this work followed standard procedures.

Bioanalyzer runs were performed with the Agilent Bioanalyzer 2100 system using the P80 kit according to

334 the manufacturer's guidelines.

Peptide mapping. $4 \mu \mathrm{g}$ of protein was diluted in $50 \%$ trifluoroethanol and further incubated at $60{ }^{\circ} \mathrm{C}$ for $2 \mathrm{~h}$ at $800 \mathrm{rpm}$. Then $2 \mathrm{mM}$ DTT was added and incubated at $37{ }^{\circ} \mathrm{C}$ for $1 \mathrm{~h}$ with shaking. The samples were diluted in $200 \mu \mathrm{L} 25 \mathrm{mM}$ ammonium bicarbonate, digested with $0.1 \mu \mathrm{g}$ trypsin at $37{ }^{\circ} \mathrm{C}$ for

341 reversed phase column (packed in-house, length $70 \mathrm{~cm}, 75 \mu \mathrm{m}$ ID, $3 \mu \mathrm{m}$ Jupiter particle from Phenomenex).

342 Peptides were separated using water/acetonitrile mobile phases with $0.1 \%$ formic acid over a gradient of $3432 \mathrm{~h}$ (ramping acetonitrile gradient $1-40 \%$ ) at a flow rate of $300 \mathrm{~nL} / \mathrm{min}$. Mass spectra of eluting peptides 344 were collected on a Thermo Q-Exactive Orbitrap. Resolution of 35k and 17.5k (at $\mathrm{m} / \mathrm{z} 200)$ were used for $345 \mathrm{MS}^{1}$ and $\mathrm{MS}^{2}$, respectively. The top 12 precursors were selected for higher energy collision (HCD) at $30 \%$ 346 normalized collision energy. A dynamic exclusion of 30s was used. Singly charged or charge unassigned 347 precursors were excluded. LCMS data were analyzed with Byonic with a semi-tryptic search of the 348 Arabidopsis thaliana protein database (source: Arabidopsis Information Resource), including common 349 contaminants. The 3XFLAG purification tag sequence was manually appended to the original PDX1.2 and 3501.3 sequences (AT3G16050.1 and AT5G01410.1). Precursor and fragment mass tolerances were set to 7 351 and $10 \mathrm{ppm}$. Protein N-terminal acetylation, pyroglutamic acid (Q), and oxidation $(\mathrm{H}, \mathrm{M}, \mathrm{W})$ were included 352 as dynamic modifications. Raw data and processing results are deposited in MassIVE (massive.ucsd.edu) 353 with accession MSV000085233. 
Intact mass determination by LCMS. $0.2-0.3 \mu \mathrm{g}$ of protein diluted in water at $0.1 \mu \mathrm{g} / \mu \mathrm{L}$ were

loaded on a Waters NanoAcquity LC equipped with a C2 reversed phase column (packed in-house, length

$70 \mathrm{~cm}, 75 \mu \mathrm{m} \mathrm{ID}, 3 \mu \mathrm{m} \mathrm{C} 2$ particle). Intact proteins were separated using water/acetonitrile mobile phases with $0.1 \%$ formic acid over a gradient of $1 \mathrm{~h}$ (ramping acetonitrile gradient 10-50\%) at a flow rate of 300

$\mathrm{nL} / \mathrm{min}$. Mass spectra of eluting proteins were collected on a Thermo VelosOrbitrap Elite. $\mathrm{MS}^{1}$ spectra were acquired at 120k resolution (at $\mathrm{m} / \mathrm{z} 200$ ) and were averaged over 8 microscans. ProMex ${ }^{33}$ was used to de-

361 convolute the data and obtain the intact mass of the proteins. LCMS data were aggregated across retention

362 time and binned into unit mass (sum intensities within $1 \mathrm{Da}$ ) to generate the intact mass plot in Fig. S5.

363 Raw data and processing results are deposited in MassIVE (massive.ucsd.edu) with accession

364 MSV000085233.

Native Mass Spectrometry (native MS). Purified PDX complexes at $0.2-0.4 \mathrm{mg} / \mathrm{mL}$

Ringoes, NJ) with gel loading pipet tips. A platinum wire connected to the electrospray voltage was inserted

371 into the capillary and was in contact with the protein solution. $1 \mathrm{kV}$ was applied at the capillary to sustain

372 the nanoelectrospray on a Thermo Q-Exactive UHMR mass spectrometer. The source was kept at $200{ }^{\circ} \mathrm{C}$,

373 with the S-lens set to $100 \%$. In-source fragmentation of $100 \mathrm{~V}$ and in-source trapping of $50 \mathrm{~V}$ were used

374 for optimum de-solvation to measure the mass of the complexes without extensive gas-phase dissociation.

375 Even with in-source fragmentation/trapping voltages off, a significant amount of PDX1.3 monomers were

376 still observed, implying that the PDX1.3 monomers were not exclusively generated by the desolvation

377 conditions. HCD gas flow was set to 2. For each final spectrum, 500 microscans were averaged. Mass

378 calibration was performed using cesium iodide clusters. To obtain the mass distribution of the PDX 12mer

379 complexes, mass spectra were processed using UniDec v2. $7^{31}$. Original data within $\mathrm{m} / \mathrm{z} 8000-11000$ were 
extracted with curved baseline subtraction, Gaussian smoothing 2.0, and binned every $3 \mathrm{~m} / \mathrm{z}$. Deconvolution was restricted to sampling mass every 10 Da with peak FWHM of 8.

Activity assay. For quantitative PLP detection, we used Enzymatic Vitamin B6 assay from A/C diagnostics (http://www.vitaminb6assay.com/). From an initial set of experiments using active enzyme PDX1.3, we determined that $5-10 \mathrm{ug} / \mathrm{ml}$ was the optimal protein concentration for conducting this assay (in place of the original design of using blood plasma) as this range results in the OD675 signal falling within the linear PLP detection range of 0-200 nmol/L (data not shown).

To compare relative enzymatic activities, all PDX samples (individual or co-expressed) were first diluted to $12.5 \mathrm{ug} / \mathrm{ml}$ concentration in TBS buffer. Then $5 \mathrm{ul}$ of $12.5 \mathrm{ug} / \mathrm{ml}$ protein were combined with 5 ul of 2XAssay buffer (50 mM Tris, pH 8.0, 1 mM ribose 5-phosphate, 2 mM D-glyceraldehyde 3-phosphate,

$39120 \mathrm{mM}$ ammonium sulfate) and incubated away from light for $30 \mathrm{~min}$ at $30^{\circ} \mathrm{C}$ in the Eppendorf 392 thermocycler. For the calibration curve, PLP (Sigma, Cat \# 82870) was diluted in a serial manner to 400 $\mathrm{nM}, 200 \mathrm{nM}, 100 \mathrm{nM}, 50 \mathrm{nM}, 25 \mathrm{nM}$ concentration in TBS buffer, further combined with an equal volume of 2XAssay buffer and incubated away from light for 30 min at $30^{\circ} \mathrm{C}$ along with PDX samples for consistency.

To quantify the amount of PLP in the mixture, $2.5 \mathrm{ul}$ of each sample were further added to the 37.5 ul of Working Binding Assay buffer (with apo-enzyme), provided by the assay kit from A/C diagnostics 398 and placed in the Corning NBS 384-well microplate. The mixture was incubated at $37^{\circ} \mathrm{C}$ for 30 min at 750 399 rpm on the Eppendorf Thermomixer C away from light. Then $20 \mathrm{ul}$ of Working Assay buffer (from the kit) 400 was added to the mixture, and incubation continued for additional $20 \mathrm{~min}$. This was followed by the addition 401 of $6.25 \mathrm{ul}$ of Chromogen RI and $3.75 \mathrm{ul}$ of Chromogen RII and $10 \mathrm{~min}$ incubation. The color development 402 was measured at $675 \mathrm{~nm}$ using the Infinite M200 PRO microplate reader by Tecan. For accuracy, all samples and calibration standards were assayed in triplicate. 
Surface induced dissocation (SID). Purified PDX complexes were buffer exchanged into $200 \mathrm{mM}$

ammonium acetate $(\mathrm{pH}$ 6.7) using the same protocol described for native MS. Then the protein solution

407 was mixed 1:1 with $100 \mathrm{mM}$ triethylammonium acetate (TEAA), and loaded into in-house pulled glass

408 capillaries (Sutter Instruments P-97 micropipet puller,with glass capillary catalog BF100-78-10). The same

409 instrument conditions were used as native MS but on a modified Thermo Q-Exactive UHMR mass

410 spectrometer as described in a previous report ${ }^{23}$. Briefly, a custom made SID cell was inserted after the

411 quadrupole and before the C-trap, replacing the original hexapole in the commercial configuration. PDX

412 complexes were mass-isolated with a $30-35 \mathrm{~m} / \mathrm{z}$ window for the $35+$ charge state, and steered towards the

413 surface under a controlled accelearation voltage of $160 \mathrm{~V}(5.6 \mathrm{keV})$. Product ions after the surface impact

414 were collected in the C-trap and mass analyzed in the Orbitrap. The electrospray voltage was set to 1.2-1.3

$415 \mathrm{kV}$, S-lens set at 100\%, in-source trapping set at -100 V, and HCD gas flow set to 4. Spectra were collected

416 at a resolution of 6,250 (at $\mathrm{m} / \mathrm{z}$ of 400 ), with an injection time of $1 \mathrm{~s}$ and averaged over 200-500 scans. Mass

417 calibration was performed using cesium iodide clusters. SID tuning voltages are listed in Table S3. SID

418 spectra were deconvoluted using UniDec v4.2.1 $1^{34}$. Mass was sampled every 10 Da with the peak FWHM

419 set to 6 , charge state smooth set at 2 and point smooth set to 1 . Experimental peaks were fit to all possible

420 combinations of PDX1.2/PDX1.3 oligomers using the mass list option in UniDec. A DScore of 30\% and a

421 peak threshold of 0.01 were used to filter the deconvolution results.

422 In the deconvoluted results (Fig. S15), peak areas were used for calculation of relative abundances

423 of different 6 mer species. The peak areas of the 6mers from both the major pathway (horizontal cleavage

424 in Fig. 3c) and the minor pathways (vertical cleavage in Fig. 3c or secondary dissociation) cannot be directly

425 distinguished by mass. We assume that the minor pathways generate different types of products with similar

426 abundances. Thus, the peak areas of the 6 mers from the minor pathways can be estimated by the median

427 peak area of the detected 4mers, 5mers, 7mers, and 8mers in the same SID spectrum. For precursor 9:3,

$42810: 2$, and 11:1, only 6mers were consistently detected and the medians were set to 0 . The peak areas of the

4296 mers from the major pathway were calculated by subtracting the contributions of minor pathways from

430 the experimtal peak areas. If the values are negative, they are reset to 0 . The "trimmed" abundances were 
normalized and plotted in Fig. 3d and Fig.S15c. The relative abundances calculated without "trimming" are

432 shown in Fig. S15d.

Cryo-EM data acquisition and processing. Quantifoil grids (658-300-AU, Ted Pella) were first glow-discharged at $15 \mathrm{~mA}$ for $1 \mathrm{~min}$ using PELCO easiGlow (Ted Pella). The grids were then transferred to a Leica EM GP the plunge-freezer, brought to $85 \%$ humidity at $25^{\circ} \mathrm{C}$ and $3 \mu 1$ of protein sample $(0.2$ $\mathrm{mg} / \mathrm{ml}$, in TBS buffer with $4 \mathrm{mM}$ DTT) was pipetted on the carbon side. The grid with sample was then collected using automated acquisition software SerialEM ${ }^{35}$. For PDX1.2 sample, 8,106 -resolution movies with 60 frames each at a dosage of 1.5 electrons per $\AA^{2}$ per frame and pixel size of $0.253 \AA$ were collected. For PDXcoexp, 6,904 super-resolution movies with 50 frames each at a dosage of 2 electrons per $\AA^{2}$ per

444 frame and pixel size of $0.253 \AA$ were acquired. All data were processed using cryoSPARC v2 software ${ }^{36}$ 445 and were corrected for full frame motion using MotionCor $2^{37}$ and CTF estimated using CTFFIND $4^{38}$. For PDX1.2 sample, a total of 787,982 particles were identified from template-based auto-picking algorithm.

447 Three rounds of reference-free 2D classification narrowed the pool to 265,224 particles. These were used 448 to generate the ab initio 3D map as a reference. Homogenous refinement in cryoSPARC v2 with D6 449 symmetry produced a final $3.2 \AA$ reconstruction. For PDXcoexp sample, a total of 510,660 particles were 450 identified from template-based auto-picking algorithm. Three rounds of reference-free 2D classification 451 narrowed the pool to 286,642 particles. These were used to generate the ab initio 3D map as a reference. 452 Homogenous refinement in cryosparc v2 with D6 symmetry produced $3.2 \AA$ reconstruction while non453 uniform refinement (BETA) yielded the $3.16 \AA$ map. Local resolution calculations on the final maps were 454 performed in Relion $3^{39}$. 
Atomic modeling. An initial homology model of PDX1.2 monomer was generated using organisms (2NV1, 5LNR, 4JDY, 2YZR and 3O07). The Cryo-EM map and the aligned initial model for a

458 single subunit were imported to PHENIX ${ }^{42}$, docked using Phenix.DockInMap, and the density region

459 surrounding the monomer unit mapped out via Phenix.MapBox. Several cycles of Phenix.RealspaceRefine

460 were carried out to refine the atomic model for the individual monomer. Map symmetry parameters were

461 then applied on the real space refined monomer model using Phenix.ApplyNCSoperators to generate the 462 12-mer, which underwent additional rounds of Phenix.RealspaceRefine. Final refinement statistics and 463 validation scores are presented in Table S1. The final models and maps were uploaded to the PDB and

EMDB databases under the accession numbers: 6PCJ and EMD-20302 for PDX1.2; and 6PCN and EMD-

20303 for PDXcoexp. Multiscale models, structural comparisons, structure-based alignments and computing of electrostatic potentials were conducted in Chimera ${ }^{43}$. Protein-protein interactions analysis was perfomed by PDBsum server ${ }^{44}$.

Rosetta calculations. Rosetta $3^{45}$ was used to characterize the features of PDX proteins (Table S2).

472 decoys ("nstruct") per each relaxation, we used the top 10\% decoys in terms of lower ("better") total score

473 values. For interface analysis and ligand binding energy calculation, we used the lowest scored decoy. For 474 interface analysis and packing status, we used InterfaceAnalyzer ${ }^{48}$ and RosettaHoles ${ }^{49}$ respectively. To 475 report feature values and analyze effectively, we used RosettaScripts ${ }^{50}$.

\section{Acknowledgements.}

478 This work was supported in part by DOE-BER Mesoscale to Molecules Bioimaging Project FWP\# 66382

479 and EMSL Strategic Science Area Projects 50188, 50165, and 50427. A portion of this research was 480 supported by NIH grant U24GM129547 and performed at the PNCC at OHSU and accessed through EMSL 
481 (grid.436923.9), a DOE Office of Science User Facility sponsored by the Office of Biological and

482 Environmental Research. CD, ZLV, and VHW acknowledge the support by the National Institutes of Health

483 Grant P41 GM128577. We thank Carrie Nicora, Karl Weitz, Anil Shukla, Ronald Moore, and Rui Zhao for

484 assistance with the LCMS experiments and Harry Scott for expert data collection at PNCC. We thank also

485 Matthew Monroe for helping with MassIVE data deposition.

\section{Author contributions.}

488 JEE, IVN and MZ devised experiments for the study. IVN performed molecular biology, cell-free 489 expression, cryoEM data processing and atomic modeling. MZ designed the all MS, native MS, and SID 490 experiments, and performed most of the related data collection and analysis. CD performed the SID data

491 collection and analysis. ZLV assisted with the SID experiments. JS assisted with the native MS setup at 492 EMSL. DNK had performed Rosetta calcualtions. MP and HH provided clones of PDX and assisted with 493 analysis of the resulting proteins. MP also performed a portion of cell-free experiments. IVN, JEE and MZ 494 wrote initial manuscript draft but all authors contributed to writing the manuscript and approval of the final 495 version.

497 Competing Interests: The authors declare no competing interests or conflicts of interest. 


\section{References:}

1. Murphy, J. M., Mace, P. D. \& Eyers, P. A. Live and let die: insights into pseudoenzyme mechanisms from structure. Curr Opin Struct Biol 47, 95-104, doi:10.1016/j.sbi.2017.07.004 (2017).

2. Yu, J. W., Jeffrey, P. D. \& Shi, Y. Mechanism of procaspase-8 activation by c-FLIPL. Proc Natl Acad Sci U S A 106, 8169-8174, doi:10.1073/pnas.0812453106 (2009).

3. Murphy, J. M. et al. The pseudokinase MLKL mediates necroptosis via a molecular switch mechanism. Immunity 39, 443-453, doi:10.1016/j.immuni.2013.06.018 (2013).

4. Schimpl, M. et al. Human YKL-39 is a pseudo-chitinase with retained chitooligosaccharidebinding properties. Biochem J 446, 149-157, doi:10.1042/BJ20120377 (2012).

5. Ribeiro, A.J. M. et al. Emerging concepts in pseudoenzyme classification, evolution, and signaling. Sci Signal 12, doi:10.1126/scisignal.aat9797 (2019).

6. Parra, M., Stahl, S. \& Hellmann, H. Vitamin B(6) and Its Role in Cell Metabolism and Physiology. Cells 7, doi:10.3390/cells7070084 (2018).

7. Mooney, S., Leuendorf, J. E., Hendrickson, C. \& Hellmann, H. Vitamin B6: a long known compound of surprising complexity. Molecules 14, 329-351, doi:10.3390/molecules 14010329 (2009).

8. Hsu, C. C. et al. Role of vitamin B6 status on antioxidant defenses, glutathione, and related enzyme activities in mice with homocysteine-induced oxidative stress. Food Nutr Res 59, 25702, doi:10.3402/fnr.v59.25702 (2015).

9. Zhang, Y. F. et al. The de novo Biosynthesis of Vitamin B6 Is Required for Disease Resistance Against Botrytis cinerea in Tomato. Mol Plant Microbe In 27, 688-699, doi:10.1094/Mpmi-01-140020-R (2014).

10. Chandrasekaran, M., Paramasivan, M. \& Chun, S. C. Bacillus subtilis CBR05 induces Vitamin B6 biosynthesis in tomato through the de novo pathway in contributing disease resistance against Xanthomonas campestris pv. vesicatoria. Sci Rep-Uk 9, doi:10.1038/s41598-019-41888-6 (2019). 
11. Tambasco-Studart, M. et al. Vitamin B6 biosynthesis in higher plants. Proc Natl Acad Sci U S A 102, 13687-13692, doi:10.1073/pnas.0506228102 (2005).

12. Leuendorf, J. E., Mooney, S. L., Chen, L. \& Hellmann, H. A. Arabidopsis thaliana PDX1.2 is critical for embryo development and heat shock tolerance. Planta 240, 137-146, doi:10.1007/s00425-014-2069-3 (2014).

13. Moccand, C. et al. The pseudoenzyme PDX1.2 boosts vitamin B6 biosynthesis under heat and oxidative stress in Arabidopsis. J Biol Chem 289, 8203-8216, doi:10.1074/jbc.M113.540526 (2014).

14. Denslow, S. A., Rueschhoff, E. E. \& Daub, M. E. Regulation of the Arabidopsis thaliana vitamin B6 biosynthesis genes by abiotic stress. Plant Physiol Biochem 45, 152-161, doi:10.1016/j.plaphy.2007.01.007 (2007).

15. Leuendorf, J. E., Osorio, S., Szewczyk, A., Fernie, A. R. \& Hellmann, H. Complex assembly and metabolic profiling of Arabidopsis thaliana plants overexpressing vitamin $\mathrm{B}(6)$ biosynthesis proteins. Mol Plant 3, 890-903, doi:10.1093/mp/ssq041 (2010).

16. Robinson, G. C. et al. Crystal structure of the pseudoenzyme PDX1.2 in complex with its cognate enzyme PDX1.3: a total eclipse. Acta Crystallogr D Struct Biol 75, 400-415, doi:10.1107/S2059798319002912 (2019).

17. Madin, K., Sawasaki, T., Ogasawara, T. \& Endo, Y. A highly efficient and robust cell-free protein synthesis system prepared from wheat embryos: plants apparently contain a suicide system directed at ribosomes. Proc Natl Acad Sci U S A 97, 559-564, doi:10.1073/pnas.97.2.559 (2000).

18. Sawasaki, T. et al. A bilayer cell-free protein synthesis system for high-throughput screening of gene products. FEBS Lett 514, 102-105 (2002).

19. Novikova, I. V. et al. Protein structural biology using cell-free platform from wheat germ. Adv Struct Chem Imaging 4, 13, doi:10.1186/s40679-018-0062-9 (2018). 
20. Titiz, O. et al. PDX1 is essential for vitamin B6 biosynthesis, development and stress tolerance in Arabidopsis. Plant J 48, 933-946, doi:10.1111/j.1365-313X.2006.02928.x (2006).

21. Wagner, S. et al. Analysis of the Arabidopsis rsr4-1/pdx1-3 mutant reveals the critical function of the PDX1 protein family in metabolism, development, and vitamin B6 biosynthesis. Plant Cell 18, 1722-1735, doi:10.1105/tpc.105.036269 (2006).

22. Mortensen, D. N. \& Williams, E. R. Electrothermal supercharging of proteins in native MS: effects of protein isoelectric point, buffer, and nanoESI-emitter tip size. Analyst 141, 5598-5606, doi:10.1039/c6an01380e (2016).

23. Leney, A. C. Subunit pI can influence protein complex dissociation characteristics. J Am Soc Mass Spectr 30, 1389-1395, doi:10.1007/s13361-019-02198-3 (2019).

24. Hall, Z., Hernandez, H., Marsh, J. A., Teichmann, S. A. \& Robinson, C. V. The role of salt bridges, charge density, and subunit flexibility in determining disassembly routes of protein complexes. Structure 21, 1325-1337, doi:10.1016/j.str.2013.06.004 (2013).

25. VanAernum, Z. L. et al. Surface-induced dissociation of noncovalent protein complexes in an extended mass range Orbitrap Mass Spectrometer. Anal Chem 91, 3611-3618, doi:10.1021/acs.analchem.8b05605 (2019).

26. Zhou, M., Dagan, S. \& Wysocki, V. H. Impact of charge state on gas-phase behaviors of noncovalent protein complexes in collision induced dissociation and surface induced dissociation. Analyst 138, 1353-1362, doi:10.1039/c2an36525a (2013).

27. Stiving, A. Q. et al. Surface-induced dissociation: an effective method for characterization of protein quaternary structure. Anal Chem 91, 190-209, doi:10.1021/acs.analchem.8b05071 (2019).

28. Harvey, S. R. et al. Relative interfacial cleavage energetics of protein complexes revealed by surface collisions. Proc Natl Acad Sci U S A 116, 8143-8148, doi:10.1073/pnas.1817632116 (2019).

29. Rodrigues, M. J. et al. Lysine relay mechanism coordinates intermediate transfer in vitamin B6 biosynthesis. Nat Chem Biol 13, 290-294, doi:10.1038/nchembio.2273 (2017). 
30. Robinson, G. C., Kaufmann, M., Roux, C. \& Fitzpatrick, T. B. Structural definition of the lysine swing in Arabidopsis thaliana PDX1: Intermediate channeling facilitating vitamin B6 biosynthesis. Proc Natl Acad Sci U S A 113, E5821-E5829, doi:10.1073/pnas.1608125113 (2016).

31. Strickler, S. S. et al. Protein stability and surface electrostatics: a charged relationship. Biochemistry-Us 45, 2761-2766, doi:10.1021/bi0600143 (2006).

32. Sawasaki, T., Hasegawa, Y., Tsuchimochi, M., Kasahara, Y. \& Endo, Y. Construction of an efficient expression vector for coupled transcription/translation in a wheat germ cell-free system. Nucleic Acids Symp Ser, 9-10 (2000).

33. Park, J. et al. Informed-Proteomics: open-source software package for top-down proteomics. Nat Methods 14, 909-914, doi:10.1038/nmeth.4388 (2017).

34. Marty, M. T. et al. Bayesian deconvolution of mass and ion mobility spectra: from binary interactions to polydisperse ensembles. Anal Chem 87, 4370-4376, doi:10.1021/acs.analchem.5b00140 (2015).

35. Mastronarde, D. N. Automated electron microscope tomography using robust prediction of specimen movements. J Struct Biol 152, 36-51, doi:10.1016/j.jsb.2005.07.007 (2005).

36. Punjani, A., Rubinstein, J. L., Fleet, D. J. \& Brubaker, M. A. cryoSPARC: algorithms for rapid unsupervised cryo-EM structure determination. Nat Methods 14, 290-296, doi:10.1038/nmeth.4169 (2017).

37. Zheng, S. Q. et al. MotionCor2: anisotropic correction of beam-induced motion for improved cryoelectron microscopy. Nat Methods 14, 331-332, doi:10.1038/nmeth.4193 (2017).

38. Rohou, A. \& Grigorieff, N. CTFFIND4: Fast and accurate defocus estimation from electron micrographs. J Struct Biol 192, 216-221, doi:10.1016/j.jsb.2015.08.008 (2015).

39. Zivanov, J. et al. New tools for automated high-resolution cryo-EM structure determination in RELION-3. Elife 7, doi:10.7554/eLife.42166 (2018).

40. Zimmermann, L. et al. A Completely Reimplemented MPI Bioinformatics Toolkit with a New HHpred Server at its Core. J Mol Biol 430, 2237-2243, doi:10.1016/j.jmb.2017.12.007 (2018). 
41. Webb, B. \& Sali, A. Protein Structure Modeling with MODELLER. Methods Mol Biol 1654, 3954, doi:10.1007/978-1-4939-7231-9_4 (2017).

42. Adams, P. D. et al. The Phenix software for automated determination of macromolecular structures. Methods 55, 94-106, doi:10.1016/j.ymeth.2011.07.005 (2011).

43. Pettersen, E. F. et al. UCSF Chimera--a visualization system for exploratory research and analysis. J Comput Chem 25, 1605-1612, doi:10.1002/jcc.20084 (2004).

44. Laskowski, R. A., Jablonska, J., Pravda, L., Varekova, R. S. \& Thornton, J. M. PDBsum: Structural summaries of PDB entries. Protein Sci 27, 129-134, doi:10.1002/pro.3289 (2018).

45. Leaver-Fay, A. et al. Rosetta3: An Object-Oriented Software Suite for the Simulation and Design of Macromolecules. Method Enzymol, 545-574, doi:10.1016/S0076-6879(11)87019-9 (2011).

46. Tyka, M. D. et al. Alternate states of proteins revealed by detailed energy landscape mapping. J Mol Biol 405, 607-618, doi:10.1016/j.jmb.2010.11.008 (2011).

47. Conway, P., Tyka, M. D., DiMaio, F., Konerding, D. E. \& Baker, D. Relaxation of backbone bond geometry improves protein energy landscape modeling. Protein Sci 23, 47-55, doi:10.1002/pro.2389 (2014).

48. Stranges, P. B. \& Kuhlman, B. A comparison of successful and failed protein interface designs highlights the challenges of designing buried hydrogen bonds. Protein Sci 22, 74-82, doi:10.1002/pro.2187 (2013).

49. Sheffler, W. \& Baker, D. RosettaHoles: rapid assessment of protein core packing for structure prediction, refinement, design, and validation. Protein Sci 18, 229-239, doi:10.1002/pro.8 (2009).

50. Fleishman, S. J. et al. RosettaScripts: a scripting language interface to the Rosetta macromolecular modeling suite. PLoS One 6, e20161, doi:10.1371/journal.pone.0020161 (2011). 

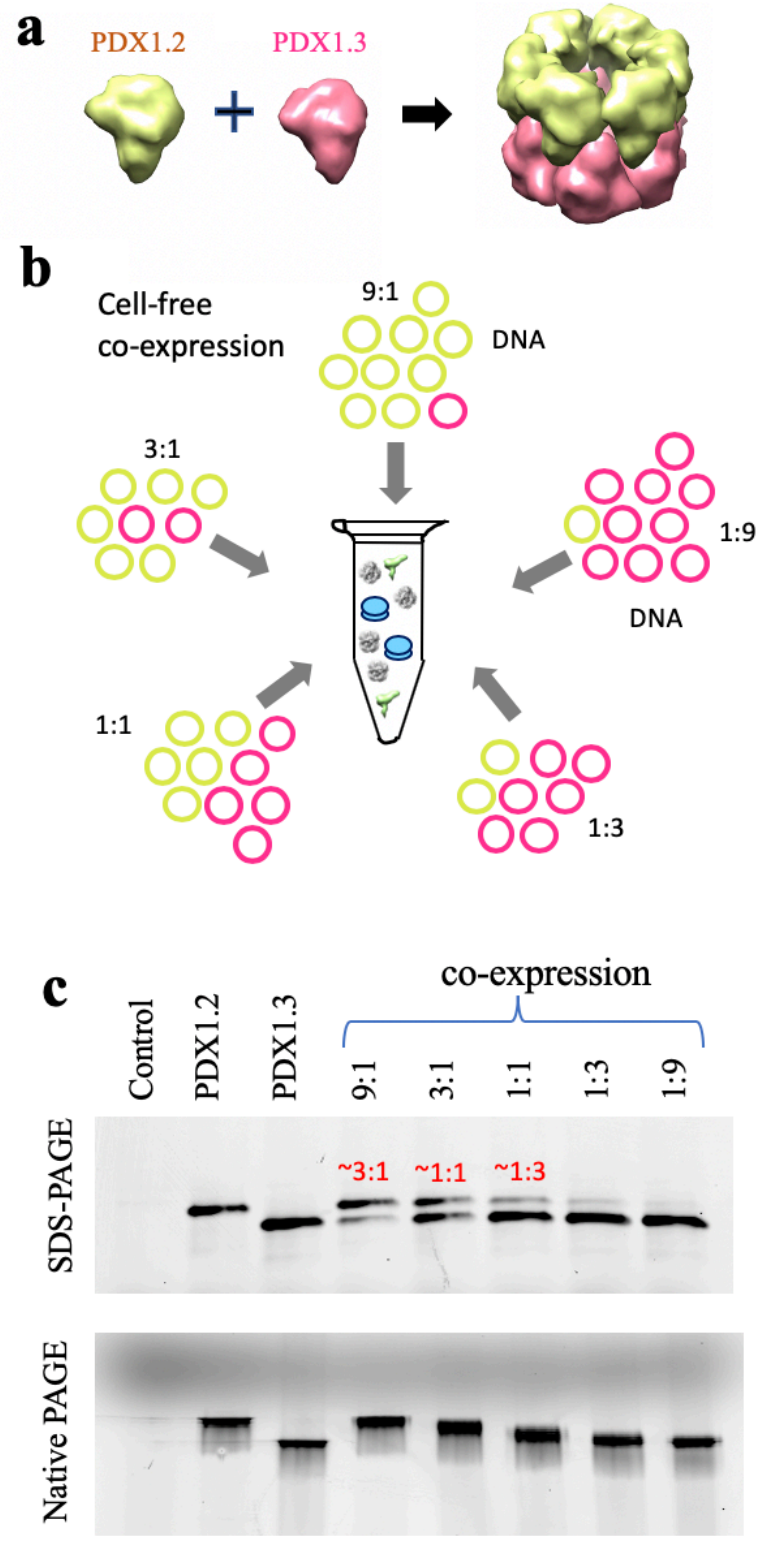

Fig. 1. PDX1.2 and PDX1.3 co-expression in a test tube. a. Schematic showing the prior proposed hetero-assembly of PDX1.2-PDX1.3 association. b. Illustration of experimental design where variable amounts of DNA plasmids are fed into the cell-free protein translation system to control and study the co-expression complexes. c. PAGE analysis of newly-synthetized PDX proteins, detected by fluorescence in a crude mixture. The control sample is a translation reaction where DNA plasmid is not supplemented. PAGE data include a denaturing SDS-PAGE gel on the top and Native PAGE on the bottom. In the co-expressed samples, protein molar ratios, determined by in- 

aCC-BY-ND 4.0 International license.

gel quantifications, are shown in red. Co-expressed conditions are denoted as 9:1, 3:1, 1:1, 1:3 and 1:9, where these numbers correspond to DNA template ratios of PDX1.2 to PDX1.3. 


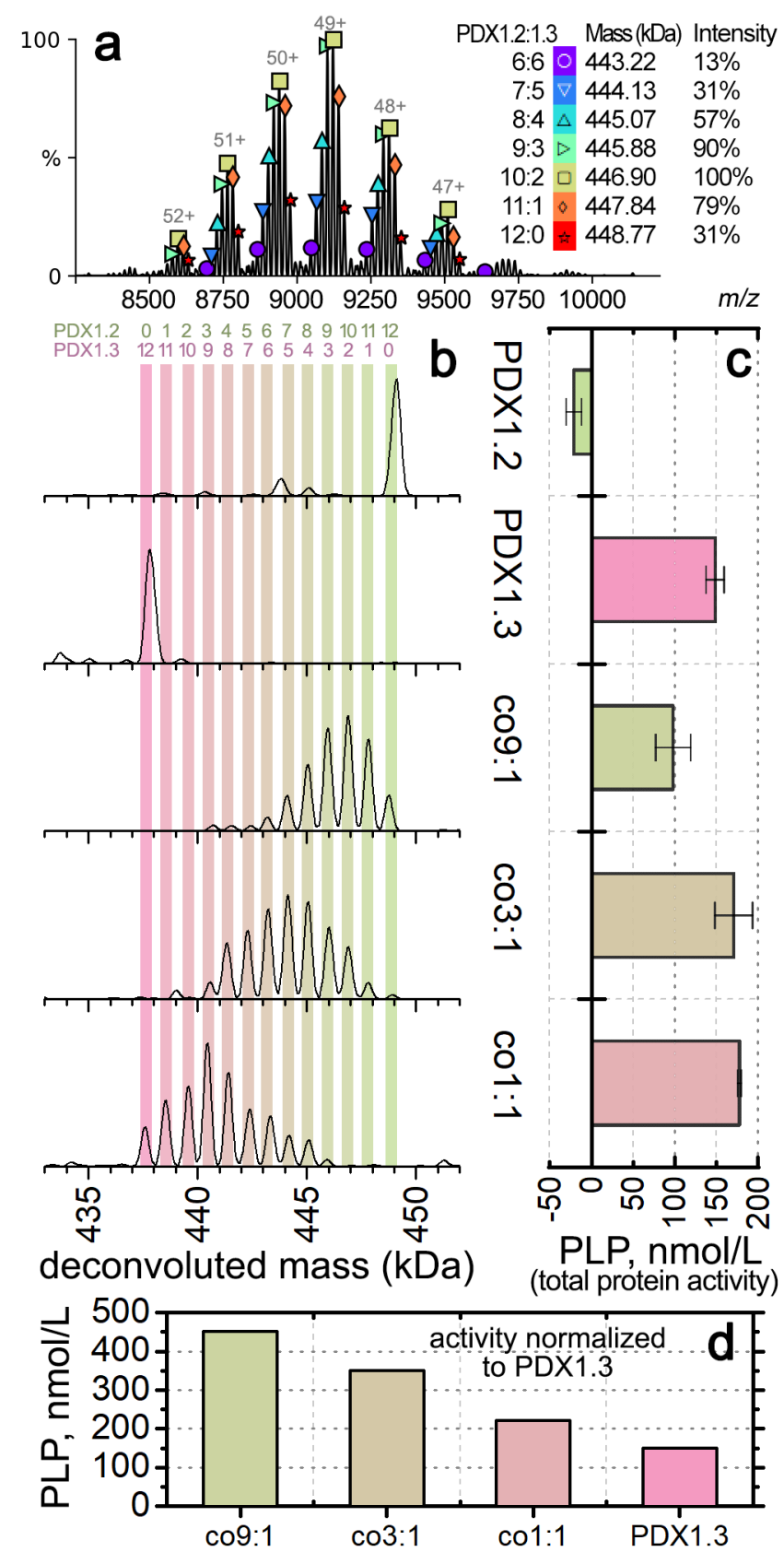

Fig.2. Oligomeric and enzymatic characterization of individual PDX homo- and hetero-complexes. a.

Representative native MS spectrum for co-expression complex 9:1, zoomed into the $12 \mathrm{mer}$ region. Each symbol above the peak indicates one 12 mer species, with their assignment, mass, and relative intensity shown on the right. Each peak with the same symbol is the same species carrying a different number of charges. Annotation was performed using UniDec. b. Deconvoluted mass distributions of PDX 12mer complexes from different samples as labeled on the right. Color bars are overlaid to show the numbers of 
PDX1.2 and PDX1.3 protomers in the PDX 12mers as noted by the numbers on the top. c. Histograms showing the enzymatic activities based on total PDX protein concentration. Error bars represent the standardard deviations from triplicate experiments. The sample names match to the mass distributions shown horizontally in (b). d. Histograms showing the enzymatic activities normalized to the amount of PDX1.3 in each sample. 


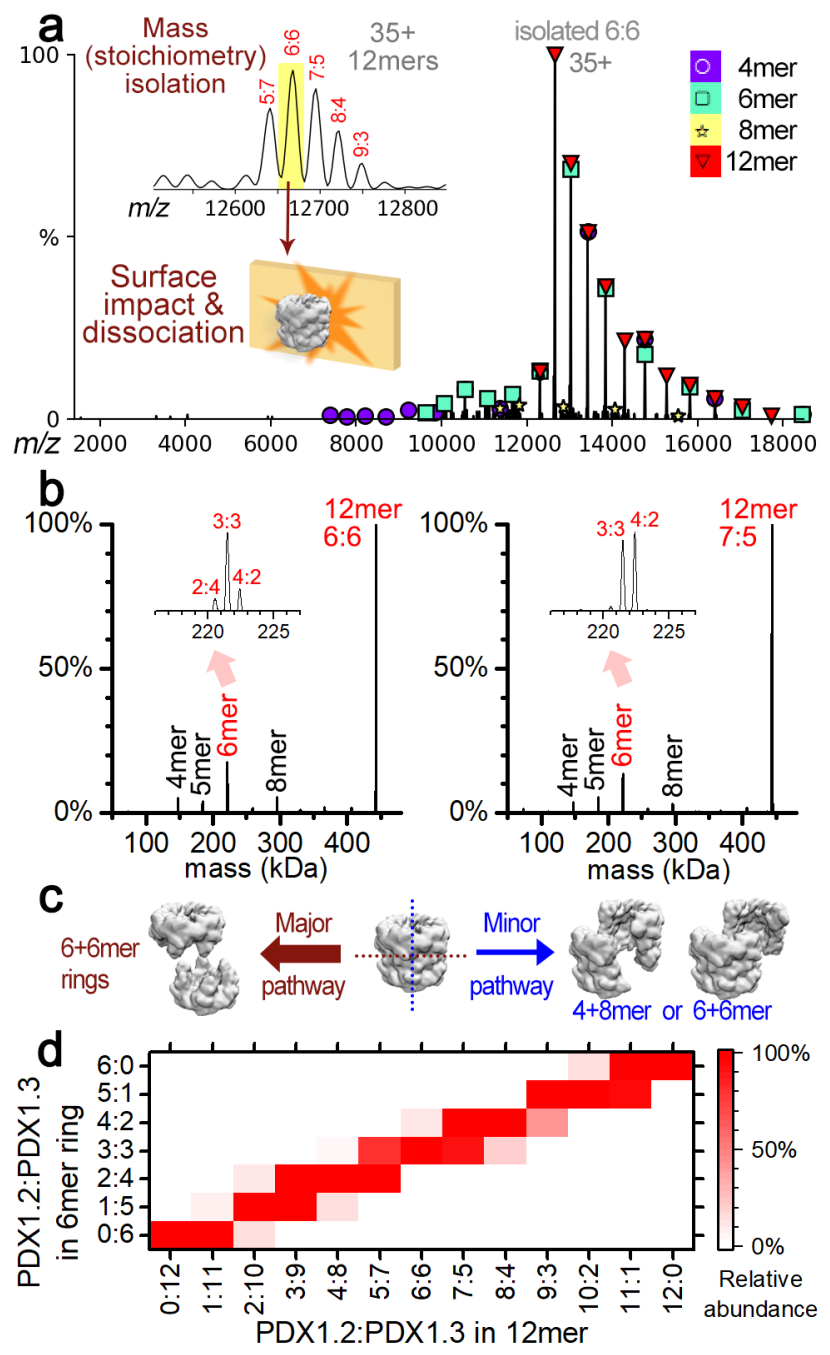

Fig.3. Dissection of PDX 12mers in the gas phase by SID. a. Representative SID spectrum for a heteromer with a single stoichiometry of 6:6 (PDX1.2:PDX1.3). Mass isolated 6:6 was collided with a surface to produce 6mers and other subcomplexes detected in the mass spectrum, which are labeled by the different symbols. The key to the symbols are on the top right. b. Deconvoluted mass distribution of the SID spectra for 6:6 (left) and 7:5 (right). Zoom-in views of the 6mer region are shown in the inserts. c. Proposed dissociation pathways for PDX by SID. The cleavage of the 12 mer in the horizonal direction is a major pathway (left). A minor pathway for cleaving in the vertical direction likely produced several other species at low abundance. d. Heatmap showing the relative abundance (normalized to max) of the released 6mer rings (vertical axis) from each stoichiometry of 12 mers (horizontal axis) following the major pathway. The 

aCC-BY-ND 4.0 International license.

contribution of 6 mers from minor pathways were estimated by the abundance of $4 / 5 / 7 / 8$ mers, and was subtracted from the raw experimental data as described in the method section. 

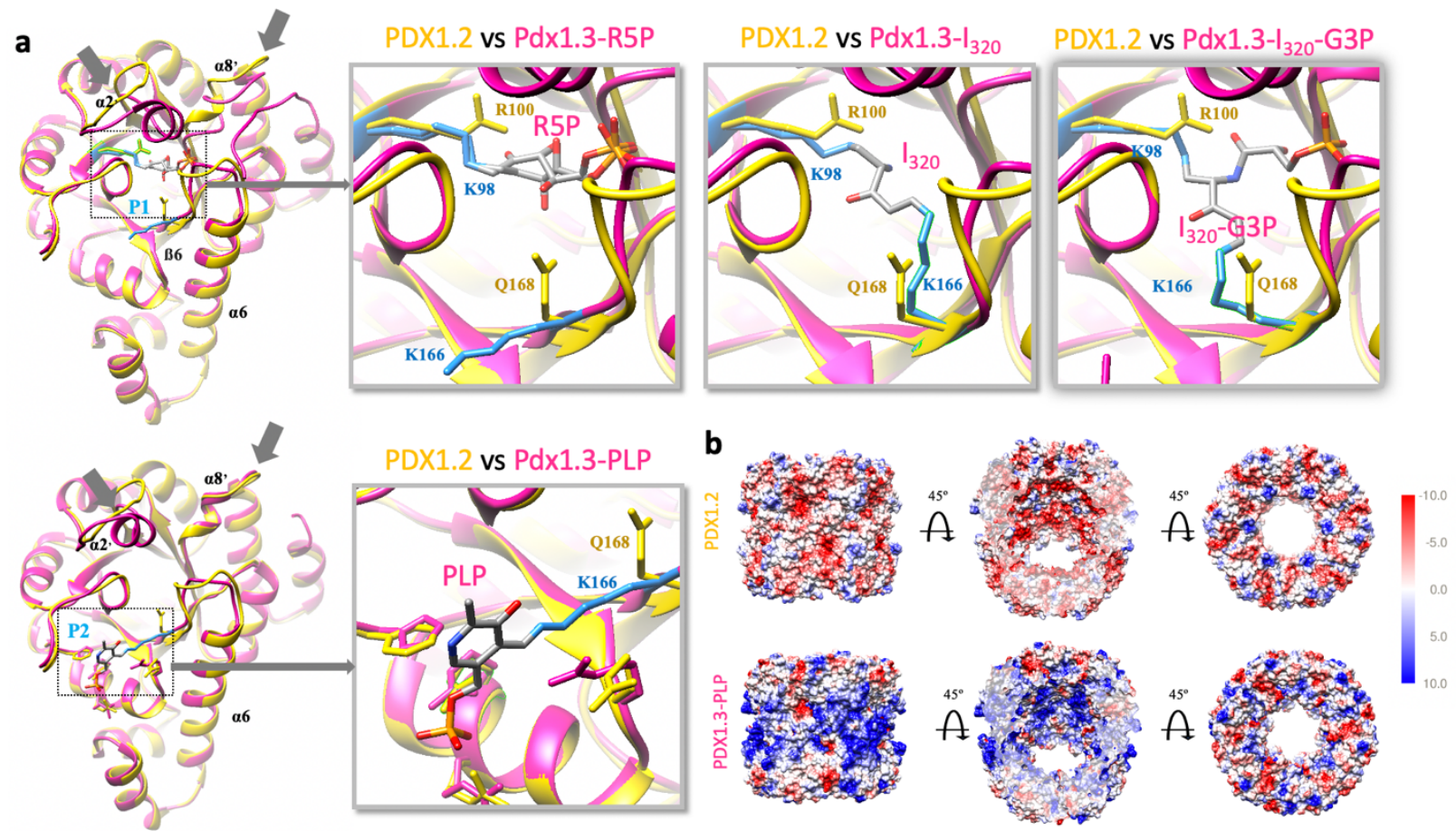

Fig.4. The cryo-EM structure of PDX1.2 versus the crystal structures of PDX1.3. a. Comparison of PDX1.2 individual subunit versus four different crystal structures, corresponding to the different stages of PLP synthesis at active sites P1 and P2. The PDX1.2 monomer subunit is in yellow, PDX1.3 subunit is in magenta. Key residues in PDX1.3 catalysis, K98 and K166, are shown in light blue. Corresponding residues in PDX1.2 include R100 and Q168 (in yellow). Substrate, intermediate products and PLP are colored by heteroatom. For structure-based sequence alignments and annotations, see Fig.S20.

b. Coulombic electrostatic potential maps. The middle images highlight the sliced view to show the interior potential of the complex. 
a

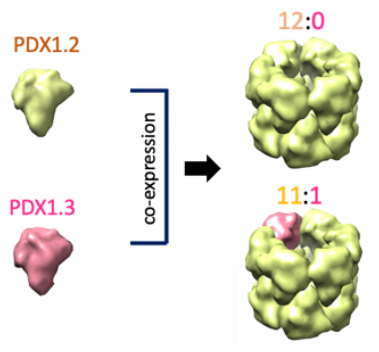

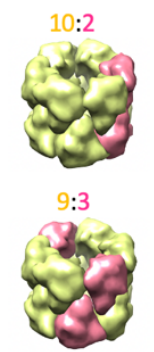

C

$\mathbf{b}$

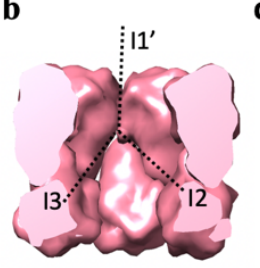

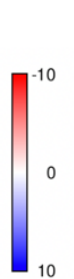

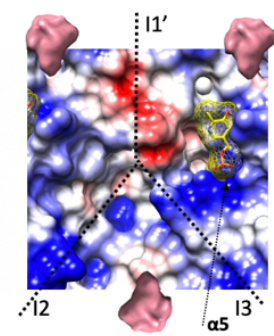

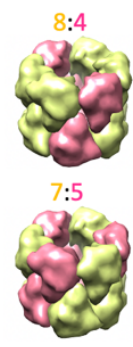
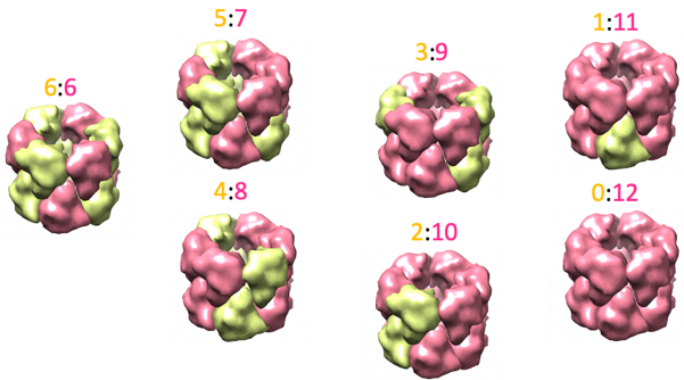

d

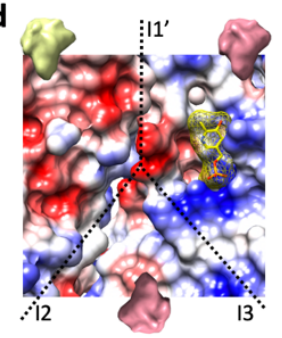

e

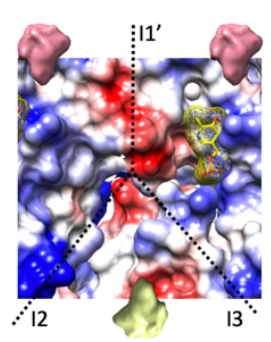

f

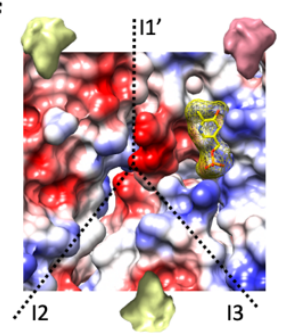

Fig.5. Heteromeric assembly mechanism and the proposed electrostatic reorganization around the P2 catalytic site. a. Revised model of PDX1.2 and PDX1.3 co-assembly. Each subclass is annotated as 12:0, $11: 1,10: 2,9: 3$ and et cetera, where the integers correspond to the number of PDX1.2 subunits relative to number of PDX1.3 subunits. Only one representative combination per class is shown while the total number of possible combinations is variable based on stoichiometry, symmetry operations and lateral favorability in the rings. b. The potential impact of PDX1.2 on PDX1.3 activity can be through I1'and I3 interfaces, surrounding the catalytic site P2 in each monomer. c. The electrostatic surface potential map of the area surrounding the P2 site in PDX1.3 homo-complex. The yellow molecule represents PLP. The models (d-f) were constructed by replacing PDX1.3 monomers with the cryo-EM structure of PDX1.2 monomers. Note the significantly changed electrostatic potential on the upper left of the P2 site in (d). 